\title{
Clasificación práctica de la gravedad y manejo médico-quirúrgico de la apendicitis aguda
}

\author{
Practical classification of gravity and medical- \\ surgical management of acute appendicitis
}

\author{
Juan Hernández-Orduña*
}

\section{Palabras clave: Apendicitis, clasificación, manejo quirúrgico, complicaciones, apéndice. \\ Keywords: Appendicitis, classification, surgical management, complications, appendix.}

* Servicio de Cirugía General, Hospital General de Atizapán, Instituto de Salud del Estado de México.

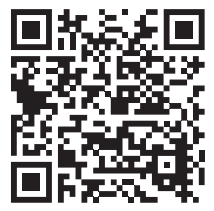

\section{RESUMEN}

Objetivo: Determinar si la reestructuración en la clasificación macroscópica de la apendicitis aguda, de acuerdo con los hallazgos, puede guiar el manejo quirúrgico, impactando directamente en la estancia hospitalaria, el pronóstico, la tasa de complicaciones y las reintervenciones. Sede: Hospital General de Atizapán, ISEM. Diseño: Estudio prospectivo, transversal, comparativo, observacional y analítico. Análisis estadístico: Se realizó estadística descriptiva de los datos demográficos, comparando los grupos de apendicitis con base en la clasificación sugerida, utilizando la t de Student para variables continuas con un intervalo de confianza de $95 \%$, y el sistema análisis de datos de Excel. La probabilidad de $<0.05$ fue aceptada como estadísticamente significativa. Material y métodos: Se estudiaron 182 pacientes que ingresaron al Servicio de Urgencias del Hospital General de Atizapán en un periodo de noviembre de 2016 a octubre de 2017, con síndrome doloroso abdominal sugestivo de apendicitis aguda; se les aplicó la clasificación sugerida en estadios evolutivos, por parte de los autores, relacionándola con el manejo sugerido y contrastándola con la clasificación tradicional y el manejo liberal de los demás cirujanos, valorando la presencia de complicaciones, reintervenciones y días de estancia hospitalaria. Resultados: Los pacientes con manejo sugerido de acuerdo con la nueva clasificación presentaron menor tasa de infección y de reintervención, comparado con los pacientes sin el manejo sugerido en la clasificación presentando una mayor tasa de infecciones $(41.1 \%)$, reintervenciones $(10.5 \%)$ y días de estancia hospitalaria (200-300\% más de estancia). Conclusiones: Esta investigación nos permite recomendar el uso de esta clasificación, ya que además de ser precisa en la gravedad de la apendicitis aguda y su relación con la contaminación de la cavidad peritoneal, da guía al manejo quirúrgico de acuerdo con los hallazgos transoperatorios, disminuyendo no sólo la tasa de complicaciones y días de estancia hospitalaria, sino también las reintervenciones.

\section{ABSTRACT}

Objective: To determine if the restructuring of the macroscopic classification of acute appendicitis according to our conclusions can guide surgical management, directly impact, in the duration of the hospital stay, in the prognosis, the rate of complications and reinterventions. Setting: General Hospital of Atizapan, ISEM. Design: Prospective, cross-sectional, comparative, observational and analytical study. Statistical analysis: Descriptive statistics applicated on the demographic data, comparing, in base of the suggested classification, the groups of appendicitis using the " $t$ " of Student for continuing variables with a 95\% confidence and Excel's data analysis system. The probability of $<0.05$ was accepted as statistically significant. Material and methods: 182 patients who entered the emergency service of the General Hospital of Atizapan in the period of time (November 2016-October 2017), with painful abdominal syndrome suggesting acute appendicitis; the suggested classification in evolutionary stages was applied to them by the authors, relating it to the suggested management and contrasting it with the traditional classification as well as the liberal management of the other surgeons, taking into consideration complications, reinterventions and hospital stay. Results: The patients with the suggested management (according to the new classification) presented lower rate of infection and reintervention in contrast to the patients without the suggested management whose infection statistics $(41.1 \%)$, reinterventions $(10.5 \%)$ and hospital stay (200-300\% more) Conclusions: This research allow us to recommend the use of the suggested classification. Being precise in the gravity of acute appendicitis and its relationship with peritoneal cavity, giving a guide for surgical management according to the trans operatory recent findings; diminishing, not only the complication rate and hospitality stay days, but also the reintervention.

Citar como: Hernández-Orduña J. Clasificación práctica de la gravedad y manejo médico-quirúrgico de la apendicitis aguda. Cir Gen. 2020; 42 (4): 263-273. https://dx.doi.org/10.35366/101395 


\section{INTRODUCCIÓN}

$\mathrm{D}_{\mathrm{a}}^{\mathrm{e}}$ e manera tradicional, la clasificación de la apendicitis aguda se ha dado en cuatro fases de acuerdo con los hallazgos transoperatorios anatomopatológicos macroscópicos; sin embargo, estos no definen claramente la gravedad de la contaminación de la cavidad peritoneal, ni su repercusión sistémica en el paciente.,

Clasificar, es decir, ordenar o disponer por clases, es la operación lógica que facilita la exposición del pensamiento en cualquier actividad y, por tanto, permite orientar la toma de decisiones. La clasificación actual de la apendicitis aguda en no perforada y perforada parece más una descripción de los hallazgos que una clasificación, la cual no nos orienta en la toma de decisiones, ya que no es lo mismo una apendicitis fase 4 sellada que una fase 4 con material purulento libre en toda la cavidad o material fecaloide, e incluso con lesión a órganos vecinos; entonces ipor qué clasificarlos y manejarlos de la misma manera? Complicaciones posteriores al manejo inicial conllevan la necesidad de estudios imagenológicos, procedimientos invasivos, mayor tiempo de manejo antibiótico y prolongación de la estancia hospitalaria con reportes de hasta un 58\% en la tasa de complicaciones, cuando la presentación inicial del cuadro es con apendicitis perforada. ${ }^{3}$

Debido a la importancia de esta patología por su alta frecuencia, es necesario identificar si la clasificación de la apendicitis aguda refleja su gravedad, guiándonos a realizar de manera adecuada la intervención quirúrgica y si esta conducta quirúrgica en cada caso repercute en las complicaciones, las reintervenciones y en la estancia hospitalaria.

El paradigma de una apendicitis aguda complicada debe cambiar, no es sólo el apéndice, ya que tenemos dos entidades: el apéndice y una cavidad peritoneal con peritonitis secundaria con las consecuencias que implica, tanto locales como sistémicas, las cuales se deben manejar en forma adecuada, sin propiciar ni esperar la peritonitis terciaria o el abdomen hostil, lo que nos puede llevar a choque séptico y muerte.

\section{Clasificaciones de apendicitis aguda}

A lo largo de los años, la clasificación de la apendicitis aguda ha sido tema de debate. La más utilizada de forma tradicional por los cirujanos se basa en la observación macroscópica de los hallazgos quirúrgicos y se divide en cuatro estadios: fase 1 eritematosa o catarral, fase 2 supurativa o flegmonosa, fase 3 necrosada y fase 4 perforada. Sin embargo, como es evidente, no define claramente la gravedad de la patología ni su repercusión en la cavidad peritoneal o sistémica. ${ }^{1,4}$ Además, esta clasificación no tiene un sustento bibliográfico, por lo que algunos autores utilizan otras: apendicitis aguda complicada o no complicada, apendicitis aguda perforada o no perforada. Lo cual demuestra que no existe un consenso unificado para su clasificación. Por lo que se han utilizado otras clasificaciones: la clínico-etiológica (no obstructiva perforada o no perforada, obstructiva perforada o no perforada, y por obstrucción vascular); evolutiva (sin perforación o con perforación, agregando peritonitis local o difusa); y topográfica de acuerdo con las variantes anatómicas de la punta apendicular. ${ }^{5}$

Maingot en el 2008 claramente define la extensión de la enfermedad, sobre todo en casos complicados, dando puerta al manejo para cada fase (1. apendicitis aguda no perforada y 2. Perforada; a) con absceso local y b) peritonitis generalizada); sin embargo, tampoco es lo mismo apendicitis aguda con fecalitos libres o sin ellos, con materia fecal libre o sin ella y en cuántos cuadrantes se encuentra o si está generalizada, además el estado de tejidos adyacentes puede ser íleo o ciego si se encuentra necrosado o con perforaciones amplias y más importante el estado sistémico del paciente. ${ }^{6}$

En el 2003, el Dr. Gilberto Guzmán clasificó la apendicitis de acuerdo con los hallazgos quirúrgicos de la siguiente manera muy parecida a la de Maingot: grado 0 sin apendicitis; la apéndice edematoso e ingurgitado; Ib apéndice abscedado o flegmonoso; Ic apéndice necrosado sin perforación; II apéndice perforado con absceso localizado; y III apendicitis complicada con peritonitis generalizada. Esta clasificación es una adaptación de la de Maingot y, al igual que él, se enfoca sólo en el apéndice sin enfatizar tejidos adyacentes y el manejo en cada caso. ${ }^{7}$

Recientemente la clasificación propuesta por la Asociación Mexicana de Cirugía General (I. Apendicitis aguda 1. No perforada: a) edematosa, hiperémica, b) abscedada, 
flegmonosa, c) necrótica. 2. Perforada: a) abscedada con peritonitis localizada, b) peritonitis generalizada. 3. Apendicitis aguda reactiva. II. Apendicitis crónica) sí define extensión de la contaminación de la cavidad; sin embargo, no de los tejidos periapendiculares o estado sistémico del paciente. Tampoco nos guía en la toma de decisión del manejo quirúrgico y postquirúrgico. $^{8}$

En el 2012, Gomes propone una clasificación de acuerdo con los hallazgos laparoscópicos en: grado 0 apéndice de aspecto normal; grado 1 hiperemia y edema; grado 2 exudado fibrinoso; grado $3 \mathrm{~A}$ necrosis segmental; grado $3 \mathrm{~B}$ necrosis de base; grado $4 \mathrm{~A}$ absceso; grado $4 \mathrm{~B}$ peritonitis regional; $\mathrm{y}$ grado 5 peritonitis difusa. ${ }^{9}$ El objetivo de este nuevo sistema fue proporcionar una clasificación estandarizada para permitir una estratificación del paciente más uniforme para la investigación de la apendicitis y ayudar a determinar el manejo óptimo según el grado. El mismo autor en 2015 da una nueva propuesta de un nuevo sistema de clasificación de apendicitis aguda basado en hallazgos clínicos, de imagen y laparoscópicos, clasificándola en: 1 ) apendicitis aguda no complicada, subdividida en grado 0 apéndice de aspecto normal (endoapendicitis/periapendicitis); grado 1 apéndice inflamado (hiperemia, edema de fibrina sin o poco líquido pericólico). 2) apendicitis aguda complicada subdividida en grado 2 necrosis, A - necrosis segmental, (sin o poco líquido pericólico), B - necrosis base, (sin o poco líquido pericólico). Grado 3 - tumor inflamatorio. A - Un Flemón. B - Absceso menos de $5 \mathrm{~cm}$ sin aire libre peritoneal. C - Absceso superior a $5 \mathrm{~cm}$ sin aire libre peritoneal, y grado 4 - perforado - peritonitis difusa con o sin aire libre peritoneal. Esta clasificación nace por la observación del autor, es necesario un nuevo sistema de clasificación integral para la apendicitis aguda, debido a que las opciones de tratamiento para los casos complicados de apendicitis aguda incluyen modalidades no operativas. $^{10}$

El porcentaje de complicaciones aumenta de acuerdo con el tipo de apendicitis aguda y su grado de evolución. Las más frecuentes son las complicaciones infecciosas, abscesos de pared y abscesos intraperitoneales, pero pueden observarse también peritonitis terciarias y abdomen hostil, lo que nos puede llevar a sepsis abdominal y choque séptico. ${ }^{11,12}$

\section{MATERIAL Y MÉTODOS}

Se realizó un estudio prospectivo, transversal, observacional y analítico en una población finita de 182 pacientes que ingresaron al Hospital General de Atizapán en el periodo comprendido entre noviembre de 2016 y octubre de 2017 con el diagnóstico de probable apendicitis aguda.

El protocolo del estudio fue aprobado por los Comités de Investigación y Ética del Hospital General de Atizapán, Estado de México. Se anexa hoja de autorización por jefatura de enseñanza e investigación, fechado el día 29 septiembre de 2016. Se respetó en todo momento la confidencialidad de la información obtenida con estricto respeto a la dignidad humana.

El presente estudio no implicó ningún riesgo para los pacientes, dado que sólo se aplicó la clasificación propuesta por el autor, correlacionándola con el hallazgo quirúrgico en relación con la fase, el manejo quirúrgico y la tasa de complicaciones, así como días de estancia hospitalaria sin influir en la decisión del Cirujano Adscrito al Servicio de Urgencias en cuanto a decisión médica o intervención quirúrgica. Se comparó el manejo realizado con el sugerido, y se contrastó de acuerdo con la evolución, complicaciones y días de estancia hospitalaria.

El objetivo de este nuevo sistema fue proporcionar una clasificación estandarizada para permitir una estratificación del paciente más uniforme para la investigación de la apendicitis, y ayudar a determinar el manejo óptimo según el estadio. Para esta finalidad, se sugirió un manejo quirúrgico de acuerdo con el estadio de la clasificación y poder comparar si guía a una conducta quirúrgica.

Se realizó estadística descriptiva de los datos demográficos, estadística analítica para comparar los grupos de apendicitis utilizando la t de Student en variables continuas para valorar la clasificación sugerida, con un intervalo de confianza de 95\%, utilizando el sistema de análisis de datos de Excel. La probabilidad de $<0.05$ fue aceptada como estadísticamente significativa. 


\section{Clasificación sugerida}

Lo importante de una clasificación es dar pauta al manejo quirúrgico, homogeneizar el tratamiento, predecir complicaciones de acuerdo con los hallazgos quirúrgicos y facilitar el proceso de sanación en la evolución del paciente, así como el proceso de enseñanza aprendizaje de los residentes de cirugía. De esta manera se puede decidir el manejo disminuyendo la prolongada estancia intrahospitalaria innecesaria.

Debemos tomar en cuenta que clasificar es disponer un conjunto de datos por clases o categorías en función de un criterio. Agrupar elementos siguiendo algún criterio, ${ }^{13}$ entonces, clasificar es la operación lógica que facilita la exposición del pensamiento en cualquier actividad y, por tanto, permite orientar la toma de decisiones. Para tratar de comprender en la apendicitis aguda el sistema de clasificación de lesiones ideal, debería estar diseñado para cumplir las siguientes condiciones:

1. Que proporcione una descripción exacta del estado de la lesión del apéndice cecal, tejidos circunvecinos y el grado de contaminación de la cavidad peritoneal.

2. Que determine el tratamiento quirúrgico más adecuado según el grado de la lesión.

3. Debe ser útil en el cálculo del pronóstico ante una posible complicación.

4. Que se ajuste a las recomendaciones del manejo quirúrgico de la apendicitis aguda complicada.

5. Que establezca normas para la prevención de infección del sitio quirúrgico, peritonitis terciaria o abdomen hostil.

Es por todo ello que se sugiere la siguiente clasificación de apendicitis aguda, correlacionándola con el manejo quirúrgico (Tabla 1). Los hallazgos son determinados por el cirujano durante el intraoperatorio y serán descritos según la clasificación utilizada.

\section{Sustento del manejo sugerido}

Uso de antimicrobianos: el tratamiento antibiótico debe comenzar desde el momento en que se realiza la indicación de la cirugía, y en la misma hora en caso de sepsis grave o de

Tabla 1: Clasificación integral de la gravedad y manejo sugerido de apendicitis aguda.

Estadio 1

Estadio 2

- Apendicitis aguda perforada con absceso localizado

Estadio 3

- Apendicitis aguda perforada con peritonitis generalizada purulenta

Estadio 4

- Apendicitis aguda perforada con peritonitis generalizada purulenta + fecalitos libres

Estadio 5

- Apendicitis aguda perforada con peritonitis generalizada y necrosis ileal o cecal

b. Abscedada

c. Necrosada

\begin{tabular}{|c|c|c|c|c|}
\hline Manejo sugerido & Manejo sugerido & Manejo sugerido & Manejo sugerido & Manejo sugerido \\
\hline $\begin{array}{l}\text { - Apendicectomía } \\
\text { y secado }\end{array}$ & $\begin{array}{l}\text { - Apendicectomía, } \\
\text { secado y drenaje }\end{array}$ & $\begin{array}{l}\text { Apendicectomía, } \\
\text { lavado de cavi- } \\
\text { dad (sin drenaje) }\end{array}$ & $\begin{array}{l}\text { - Apendicectomía + lava- } \\
\text { do exhaustivo y nueva } \\
\text { laparatomía sistemática } \\
\text { en } 48 \text { horas conforme a } \\
\text { los criterios de interven- } \\
\text { ción }\end{array}$ & $\begin{array}{l}\text { - Apendicectomía + hemi- } \\
\text { colectomía derecha con/o } \\
\text { desfuncionalización ileal } \\
\text { + lavado exhaustivo y } \\
\text { nueva LAPE sistemática } \\
\text { en } 48 \text { horas de acurdo a } \\
\text { criterios de reintervención }\end{array}$ \\
\hline 1 & 2 & 3 & 4 & 5 \\
\hline
\end{tabular}

Fuente: Elaboración propia. 
choque séptico. ${ }^{2,14}$ En las primeras horas de tratamiento, el objetivo de la antibioticoterapia es limitar las bacteriemias y reducir la frecuencia de abscesos residuales. ${ }^{2,15} \mathrm{El}$ manejo antibiótico debe cubrir, idealmente, microorganismos aerobios y anaerobios. Después, conforme a las guías, toma de cultivo para poder dirigir la terapia. El uso de antimicrobianos debe de continuarse con manejo por vía intravenosa hasta transcurrir un mínimo de 24 horas sin fiebre ni leucocitosis, agregando a esto estado general adecuado del paciente y función intestinal reestablecida. ${ }^{4}$

Tratamiento quirúrgico: la antibioticoterapia contribuye a mejorar el pronóstico, ${ }^{15}$ pero no es suficiente para conseguir la curación. Es fundamental un procedimiento quirúrgico adecuado para controlar el origen de la infección. El objetivo fundamental en el tratamiento quirúrgico de la apendicitis aguda complicada con peritonitis secundaria es el control de la causa de la contaminación peritoneal y la prevención de una sepsis residual.

Drenaje de la zona quirúrgica: los drenajes se deben utilizar para drenar un absceso no drenado, drenado en forma insuficiente o para establecer una fistula controlada. Fuera de estas indicaciones, la colocación de drenajes es ineficaz en la peritonitis generalizada, ya que la fibrina rodea con rapidez los drenajes a lo largo de su trayecto intraperitoneal y bloquea su efecto de salida en forma efectiva. ${ }^{1}$ No es necesario usar drenajes si se realizó una limpieza adecuada de la cavidad abdominal. ${ }^{8}$

Lavado peritoneal transoperatorio en peritonitis generalizadas: la reducción del inóculo bacteriano en la cavidad abdominal se obtiene mediante la aspiración del material purulento, exploración de los fondos de saco y correderas parietocólicas, así como de los espacios subfrénicos y subhepática. El lavado transoperatorio con solución salina tibia es un procedimiento generalmente realizado durante la laparotomía por peritonitis difusa, la adición de antibióticos al lavado no parece influir en la evolución del cuadro de contaminación intraabdominal. ${ }^{16}$

El lavado de toda cavidad abdominal debe realizarse hasta que el líquido se encuentre claro, con lo que se obtiene excelente supervivencia y mínima sepsis residual en pacientes con peritonitis grave. La cantidad necesaria de líquido de lavado peritoneal varía según el paciente; en algunos casos puede alcanzar de 10 a 15 litros en las peritonitis postoperatorias graves estercoraceas. ${ }^{17,18}$

En la actualidad, ya no se utilizan tantos litros para el lavado, sólo de 3 a 5 litros, debido a otros estudios que, si bien fueron realizados en animales, se han tomado como pauta, los cuales mencionan que hay que tener en cuenta que el lavado peritoneal altera los mecanismos locales de defensa de la cavidad abdominal; la solución salina actúa como coadyuvante para alterar la fagocitosis y migración de leucocitos en la cavidad abdominal, ${ }^{19}$ la adición de antibióticos o antisépticos al líquido de lavado altera la quimiotaxis de los neutrófilos, inhibe su actividad microbicida ${ }^{20}$ y aumenta la formación de adherencias. ${ }^{21}$

Luego del lavado es importante un secado estricto de la cavidad abdominal, pues la solución salina residual diluye las opsoninas bacterianas, deja a las bacterias en suspensión en un medio líquido, reduce la fagocitosis y permite la proliferación bacteriana.

Recientemente se ha escrito del uso de solución electrolizada por selectividad iónica para lavado peritoneal transoperatorio. Se utilizaron entre 6 a 10 litros de la solución correspondiente, previamente calentada a $37^{\circ} \mathrm{C}$ hasta eliminar por completo la contaminación macroscópica. ${ }^{22}$ Nosotros no contamos con experiencia con estas soluciones.

Reintervenciones: en las infecciones más graves, en particular en las infecciones postoperatorias, no existe ningún medio de drenar eficazmente toda la cavidad peritoneal. ${ }^{23}$ Se han propuesto dos actitudes: las reintervenciones a demanda, que no aportan una satisfacción completa debido al retraso de la revisión que se observa a veces en pacientes complejos, y las reintervenciones sistemáticas programadas cada 24-48 horas hasta conseguir una cavidad peritoneal macroscópicamente limpia. ${ }^{24}$ Los criterios de reintervención son condicionales (Tabla 2). El cumplimiento estricto de los criterios formales de reintervención permite actuar antes de que se produzcan accidentes cardiocirculatorios secundarios a choque tóxico-infeccioso. Las primeras manifestaciones de la infección peritoneal, secundarias a la 
pérdida de hermeticidad del cierre del muñón apendicular o una lesión incidental, al igual que en peritonitis terciaria, son muy precoces, casi siempre dentro de los tres días siguientes a la operación. La primera manifestación es con mayor frecuencia la fiebre, le siguen diarrea, hipersecreción y estasis gástrica, hipo, taquicardia aislada, disminución importante de la diuresis y, por último, falta de reanudación del tránsito intestinal o interrupción secundaria. ${ }^{17}$

Conducta ante la lesión causal: en ocasiones, se descubre una necrosis cecal adyacente, una lesión de la última porción del intestino delgado, o ambas; en cualquier caso, el tratamiento consiste en resección ileocólica con ileostomía y colostomía terminal. En un paciente en estado de choque, la perfusión tisular, incluida la intestinal, está alterada. En estas circunstancias, el riesgo de dehiscencia de una sutura de nueva realización es elevado. Existe el mismo riesgo de dehiscencia de sutura tras la realización de una anastomosis digestiva en un entorno séptico. Por lo tanto, parece prudente en dichas situaciones renunciar a establecer de inmediato la continuidad digestiva en favor de las ostomías. ${ }^{15}$

Prevención de sepsis residual e infección del sitio quirúrgico: para prevenir la sepsis residual de un paciente intervenido por un cuadro de apendicitis complicada con peritonitis generalizada, en especial la formación de abscesos residuales, debe tenerse como objetivo fundamental ayudar a los mecanismos de defensa del peritoneo a recuperar una función normal. Desde la intervención inicial se debe efectuar en forma muy cuidadosa una limpieza y debridación de todo el material fibrinopurulento que se encuentre.

Por otro lado, se ha mencionado como medida general para prevenir la infección del sitio quirúrgico (ISQ) la remoción del vello abdominal, que debe ser inmediatamente antes de la operación. ${ }^{25}$ La preparación apropiada de la piel al momento del procedimiento operatorio con un agente antiséptico es una medida preventiva bien establecida. ${ }^{26}$ La manipulación

Tabla 2: Criterios de reintervención en peritonitis.

\begin{tabular}{|c|c|c|c|}
\hline $\begin{array}{l}\text { Criterios para conducta } \\
\text { conservadora }\end{array}$ & $\begin{array}{l}\text { Criterios par conducta } \\
\text { conservadora }\end{array}$ & $\begin{array}{l}\text { Criterios de } \\
\text { reintervención rápida }\end{array}$ & $\begin{array}{l}\text { Criterios de } \\
\text { reintervención rápida }\end{array}$ \\
\hline $\begin{array}{l}\text { - Diuresis conservada } \\
\text { (+ de } 40 \text { ml/hora) } \\
\text { - Estado cardiocirculatorio } \\
\text { estable sin necesidad de } \\
\text { recurrir en forma prolon- } \\
\text { gada a aminas vasopreso- } \\
\text { ras y, sobre todo, sin tener } \\
\text { que aumentar progresiva- } \\
\text { mente las dosis } \\
\text { - Falta de signos toxiinfec- } \\
\text { ciosos generales } \\
\text { - Falta de signos abdomi- } \\
\text { nales de difusión; tránsito } \\
\text { conservado o restablecido } \\
\text { y disminución de la salida } \\
\text { de líquido por la sonda } \\
\text { nasogástrica }\end{array}$ & $\begin{array}{l}\text { - Rápida desaparición del } \\
\text { signo de alarma que llevó a } \\
\text { sospechar el diagnóstico de } \\
\text { peritonitis postoperatoria } \\
\text { - Polimorfonucleares neu- } \\
\text { trófilos ligeramente ele- } \\
\text { vados o, en caso de clara } \\
\text { elevación, caída acentuada } \\
\text { de los valores con respecto } \\
\text { a la cifra precedente } \\
\text { - Insuficiencia renal funcio- } \\
\text { nal fácilmente corregible } \\
\text { - Falta de indicación de } \\
\text { ventilación asistida o } \\
\text { de prolongación de la } \\
\text { misma en un paciente sin } \\
\text { insuficiencia respiratoria } \\
\text { preoperatoria }\end{array}$ & $\begin{array}{l}\text { - Oligoanuria } \\
\text { - Estado cardiocirculatorio } \\
\text { insuficiente con deterioro } \\
\text { creciente } \\
\text { - Falta de respuesta clínica } \\
\text { y de laboratorio satisfacto- } \\
\text { ria al tratamiento médico } \\
\text { intensivo } \\
\text { - Signos abdominales de } \\
\text { propagación; falta de } \\
\text { reanudación del tránsito } \\
\text { intestinal o detención } \\
\text { secundaria }\end{array}$ & $\begin{array}{l}\text { - Gradiente elevado de } \\
\text { leucocitosis } \\
\text { - Persistencia de la insu- } \\
\text { ficiencia renal a pesar } \\
\text { del tratamiento médico } \\
\text { intensivo, o agravación de } \\
\text { la insuficiencia renal } \\
\text { - Necesidad de ventilación } \\
\text { asistida }\end{array}$ \\
\hline
\end{tabular}

Fuente: Elaboración propia como resultado de la investigación.

Adaptada de tratado de técnicas quirúrgicas digestivas, Elsevier/Mason 17. 
gentil de los tejidos, protección de bordes de la herida con compresas, limpieza completa de la contaminación, remoción completa de los tejidos necróticos o desvitalizados y evitar los espacios muertos son importantes para evitar la infección. Por último, evitar la hipotermia, mantener altas concentraciones de oxígeno en los tejidos y evitar la hiperglucemia han sido mencionados en relación con la prevención de la ISQ. ${ }^{27,28}$

\section{Aplicación del manejo sugerido de acuerdo con el estadio}

A. Estadio 1: Existe escasa presencia de bacterias en el líquido periapendicular, por lo que el tratamiento sugerido es de apendicectomía, secado de cavidad y manejo antimicrobiano por dos días de antibióticos intravenosos (IV). En nuestro hospital utilizamos en primera instancia metronidazol 500 mg IV cada ocho horas o clindamicina $600 \mathrm{mg}$ IV cada ocho horas, y en conjunto con ceftriaxona o cefotaxima $1 \mathrm{~g}$ IV cada 12 horas. En niños se calcula dosis de cada antibiótico conforme a su peso. El uso de antimicrobianos debe continuarse con manejo por vía intravenosa hasta cursar un mínimo de 24 horas sin fiebre ni leucocitosis, agregando a esto estado general adecuado del paciente y función intestinal reestablecida. ${ }^{4}$

B. Estadio 2: Apéndice perforado con absceso localizado. El tratamiento sugerido es apendicectomía, secado y drenaje en área cruenta y antimicrobianos tres a cuatro días IV.

C. Estadio 3: Apendicitis perforada con peritonitis generalizada purulenta. El tratamiento sugerido apendicectomía y lavado de la cavidad peritoneal sin drenajes y antimicrobianos IV por cinco a siete días (ver drenaje de zona quirúrgica en sustento de manejo).

D. Estadio 4: Apéndice perforado con peritonitis generalizada purulenta y fecalitos libres. Tratamiento de apendicectomía con lavado exhaustivo de cavidad peritoneal sin drenaje, con reoperación a las 48 horas sistematizada para nuevo lavado conforme a los criterios de reintervención y evolución clínica (ver drenaje de zona quirúrgica).

E. Estadio 5: Apéndice perforado en estadio 4 más necrosis de íleon o ciego. Tratamien- to: hemicolectomía derecha con lavado de cavidad e ileostomía o anastomosis ileocolónica (con base en contaminación de cavidad y si presenta choque séptico o no). Con lavado de cavidad peritoneal sin drenaje, con reoperación a las 48 horas sistematizada para nuevo lavado conforme a los criterios de reintervención y evolución clínica (ver drenaje de zona quirúrgica).

\section{RESULTADOS}

Todos los resultados estadísticos descriptivos se presentaron en el artículo de nuestra autoría, ya que fue un estudio doble, el de escala diagnóstica y al mismo tiempo el de la clasificación. ${ }^{29}$

De los 182 casos revisados, 110 fueron hombres y 72 mujeres, y una relación masculina/femenino de 1.5/1. Con mayor frecuencia de edades entre 10 y 25 años.

El tiempo de evolución del cuadro clínico antes de ingresar al hospital fue de dos a tres días en promedio, excepto en casos complicados que llevó en promedio 15 días.

El síntoma más común fue el dolor en la fosa iliaca derecha, migración del dolor (90\%), náusea o vómito (90\%). El síntoma menos común fue anorexia (18\%). Los signos más comúnmente detectados fueron: McBurney (98.6\%) y Von Blumberg (90\%).

Se presentaron las siguientes complicaciones postquirúrgicas: 19\% con infección del sitio operatorio, reintervención en nueve casos por infección de sitio operatorio de tipo órgano-espacio (clasificación ya establecida de infección del sitio quirúrgico que la divide en superficial, profunda y órgano espacio con presencia de absceso, ya sea en fascias superficiales, profundas o en cavidad). Certificado con ultrasonido (USG) con evidencia de absceso residual, más estado clínico de fiebre, distención y diarrea en despeñe. Dos casos requirieron hemicolectomía y estoma con tiempo de evolución de 15-20 días y presencia de necrosis y perforación de ciego.

De los pacientes, $8.7 \%$ no fueron diagnosticados por patología con apendicitis, pero los cuadros más complicados con mayor tiempo de evolución por no realizarse el diagnóstico temprano fueron de $26.37 \%$, siendo más frecuentes en casos de niños, mujeres y ancianos, 
lo que llevó a más días de estancia hospitalaria, complicaciones quirúrgicas y postquirúrgicas, así como a costo hospitalario más elevado.

De los 182 casos revisados en relación con la fase apendicular corroborada por estudio histopatológico, observamos mayor frecuencia de fase 4 (estadio 2). Se presentaron las siguientes complicaciones postquirúrgicas: 34 casos (19\%) con infección del sitio operatorio, reintervención en nueve casos (4.9\%) por infección de sitio operatorio de tipo órgano-espacio (clasificación ya establecida de infección del sitio quirúrgico que la divide en superficial, profunda y órgano espacio con presencia de absceso, ya sea en fascias superficiales, profundas o en cavidad). ${ }^{30}$ Dos casos (1.09\%) con tiempo de evolución prolongado de 15-20 días, lo que llevó a necrosis y perforación de ciego, requirieron hemicolectomía y estoma.

En relación con la clasificación propuesta y su relación directa con el manejo quirúrgico sugerido, los datos se muestran en la Tabla 3 y Figuras 1 a 3, de las cuales desprendemos primero que de los 81 casos en estadio uno, la tasa de complicación y reintervención es muy baja o nula. Para el estadio dos, la tasa de infecciones es muy baja con el manejo sugerido con sólo un caso de infección de sitio

\begin{tabular}{lcccc}
\multicolumn{5}{c}{$\begin{array}{c}\text { Tabla 3: Relación de casos de apendicitis aguda por } \\
\text { estadio, complicaciones y días de estancia hospitalaria } \\
\text { con base en la clasificación sugerida y su manejo. }\end{array}$} \\
& $\begin{array}{c}\text { Estadio/ } \\
\text { pacientes }\end{array}$ & $\begin{array}{c}\text { Complica- } \\
\text { ciones }\end{array}$ & $\begin{array}{c}\text { Reinterven- } \\
\text { ciones }\end{array}$ & $\begin{array}{c}\text { Días de } \\
\text { estancia }\end{array}$ \\
\hline Pacientes & $1 / 81$ & 0 & 0 & 3 \\
con manejo & $2 / 46$ & 1 & 0 & 6 \\
sugerido & $3 / 3$ & 0 & 0 & 5 \\
& $4 / 0$ & 0 & 0 & 0 \\
Pacientes & $5 / 0$ & 0 & 0 & 0 \\
sin manejo & $1 / 0$ & 0 & 0 & 0 \\
sugerido & $2 / 25$ & 25 & 2 & $7-10$ \\
& $3 / 5$ & 5 & 3 & $12-18$ \\
& $4 / 4$ & 2 & 2 & $15-20$ \\
p & $5 / 2$ & 2 & 2 & $15-20$ \\
& & 0.22664 & 0.021312 & 0.044950 \\
\hline
\end{tabular}

Fuente: Elaboración propia como resultado de la investigación.

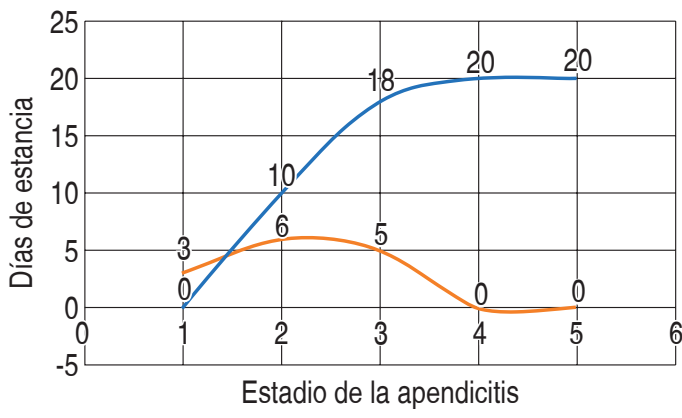

- Días de estancia con manejo sugerido

- Días de estancia sin manejo sugerido

\begin{tabular}{llc}
\multicolumn{3}{c}{$\begin{array}{c}\text { Pruebas t para dos muestras, días de } \\
\text { estancia en apendicitis aguda. }\end{array}$} \\
\hline & Con manejo & Sin manejo \\
& sugerido & sugerido \\
\hline Media & 2.8 & 13.6 \\
Varianza & 7.7 & 74.8 \\
Observaciones & 5 & 5 \\
Diferencia hipotética de las medias & 0 & \\
Grados de libertad & 5 & \\
Estadístico t & -2.65877621 & \\
p (test) una cola & 0.02247496 & \\
Valor crítico de t (una cola) & 2.01504837 & \\
p (test) dos colas & 0.044950 & \\
Valor crítico de t (dos colas) & 2.57058184 &
\end{tabular}

Figura 1: Días de estancia de pacientes con apendicitis aguda en estadio 1, 2, 3, 4 y 5 con manejo sugerido y sin manejo sugerido conforme a la clasificación propuesta. Fuente: Elaboración propia como resultado de la investigación.

quirúrgico que requirió curaciones y seis días de estancia; comparada con los 24 casos, cuando el manejo no es el sugerido. Dos casos requirieron reintervención por infección de órgano espacio, con hallazgos de abscesos interasa, pélvico y subfrénico derecho. En este estadio tenemos un $47.8 \%$ de complicados de los 71 casos, y $13 \%$ del total de 182 casos. Este $47.8 \%$ de casos complicados se presentó más en los pacientes sin el manejo sugerido de acuerdo con el estadio y requirió más días de estancia hospitalaria, de siete a 10 días, para curaciones y mayor tiempo de antibióticos. Por otro lado, aunque sólo tuvimos dos reintervenciones, se presentaron en los que no se realizó el manejo sugerido y la estancia hospitalaria se elevó hasta 12-15 días con el consiguiente gasto hospitalario en todos los rubros: dos cirugías, más días 
en cama, antibióticos, soluciones intravenosas, horas hombre, etcétera.

En estadio 3, los cinco casos sin manejo sugerido presentaron complicación de infección de sitio quirúrgico superficial y profundo, en tres casos se requirió reintervención por absceso residual y respuesta inflamatoria sistémica, con más días de estancia hospitalaria llegando a 1218 días con mayor gasto hospitalario en general.

En estadio 4 y 5, en donde no se realizó el manejo sugerido, se encontraron cuatro casos que requirieron reintervención por infección de órgano espacio, estancia hospitalaria de 15 a 20 días con un alto gasto y el riesgo de mayor morbimortalidad para el paciente.

En resumen, tuvimos 85 casos en estadio 2-5 (lo que sería fase IV), de los cuales, 35 casos $(41.1 \%)$ presentaron complicaciones y no fueron estadificados como peritonitis secundaria a apendicitis aguda complicada ni fueron manejados en forma sugerida. En nueve casos $(10.5 \%)$ se requirió de reintervención, con un aumento en el costo hospitalario y sobre todo mayor riesgo de morbimortalidad. Con una estancia hospitalaria de 15 a 20 días, lo que nos da un $200-300 \%$ más que los casos no complicados (tres a cinco días). Sin contar que está pendiente su reconexión de tránsito

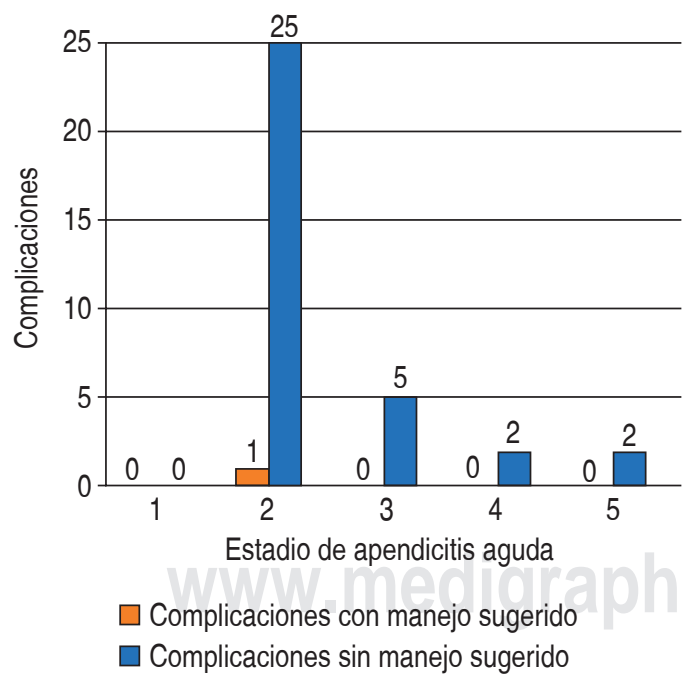

Figura 2: Tasa de complicaciones de pacientes con apendicitis aguda en estadio 1, 2, 3, 4 y 5 con manejo sugerido y sin manejo sugerido de acuerdo con la clasificación propuesta.

Fuente: Elaboración propia como resultado de la investigación.

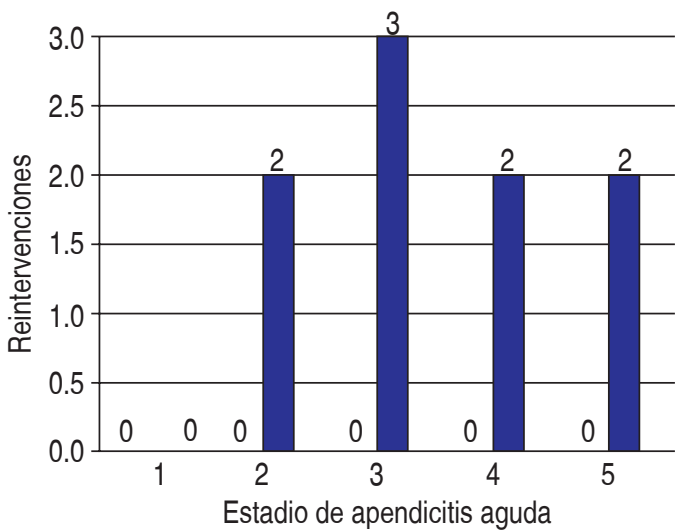

Reintervenciones sin manejo sugerido

$\square$ Reintervenciones con manejo sugerido

\begin{tabular}{|lcc}
\hline \multicolumn{2}{|c}{$\begin{array}{c}\text { Pruebas t para dos muestras reintervenciones } \\
\text { de pacientes con apendicitis aguda. }\end{array}$} \\
\hline & $\begin{array}{c}\text { Con manejo } \\
\text { sugerido }\end{array}$ & $\begin{array}{c}\text { Sin manejo } \\
\text { sugerido }\end{array}$ \\
\hline Media & 0 & 1.8 \\
Varianza & 0 & 1.2 \\
Observaciones & 5 & 5 \\
Diferencia hipotética de las medias & 0 & \\
Grados de libertad & 4 & \\
Estadístico t & -3.67423461 & \\
p (test) una cola & 0.01065582 & \\
Valor crítico de t (una cola) & 2.13184679 & \\
p (test) dos colas & 0.021312 & \\
Valor crítico de t (dos colas) & 2.77644511 \\
\hline
\end{tabular}

Figura 3: Tasa de reintervenciones de pacientes con apendicitis aguda en estadio 1, 2, 3, 4 y 5 con manejo sugerido y sin manejo sugerido conforme a la clasificación propuesta.

Fuente: Elaboración propia como resultado de la investigación.

intestinal, que nos llevará más días de estancia hospitalaria.

\section{DISCUSIÓN}

En relación con la clasificación sugerida, no encontramos mucha literatura, sólo cuatro artículos que sugieren una nueva clasificación, ya contemplando que no es adecuada la clasificación macroscópica sin relacionar la gravedad con el manejo quirúrgico.

Gilberto Guzmán-Valdivia en $2003^{7}$ sugiere una clasificación útil en la apendicitis aguda 
dividiendo en 3 grados desde apendicitis no perforada hasta la perforada con peritonitis generalizada, sugiriendo a la vez el manejo quirúrgico con resultados importantes en la tasa de complicaciones y estancia hospitalaria. Sin embargo, no toma en cuenta o no define si la contaminación peritoneal es purulenta o fecaloide y si está lesionado el ciego o íleo, por lo cual consideramos nuestra clasificación más específica y completa.

Por otro lado, en 2015, Sergio David Castañeda ${ }^{3}$ sugiere cambio de la clasificación macroscópica de la apendicitis aguda, valorando si tiene alguna influencia en la duración de la estancia hospitalaria y la tasa de complicaciones dado el cambio en el manejo antibiótico postoperatorio. Observó una disminución en el número de días de hospitalización/año y en el número de dosis de antibiótico/año y reducción en el número de complicaciones. Concluyendo que el cambio en la clasificación macroscópica y el aportar la nueva definición sobre apendicitis perforada ha logrado disminuir la estancia hospitalaria y la cantidad de antibióticos utilizados sin una repercusión significativa en la tasa de complicaciones, dando mayor énfasis en el uso de antibióticos. En nuestra clasificación, vamos más allá del uso de antibióticos con prevención de infección del sitio quirúrgico, peritonitis terciaria y abdomen hostil.

En el 2012, Gomes ${ }^{9}$ propone una clasificación de acuerdo con los hallazgos laparoscópicos; sin embargo, está limitado por su enfoque exclusivo en los aspectos intraoperatorios, es decir, continua con descripción macroscópica, sólo que vista por laparoscopia. El mismo autor en $2015^{10}$ da una nueva propuesta de un nuevo sistema de clasificación de apendicitis aguda basado en hallazgos clínicos, de imagen y laparoscópicos sin hablar de grado de contaminación de cavidad peritoneal y su manejo, enfocándose en manejo con antibióticos en etapas tempranas, con tres días de manejo y en avanzados o complicados hasta 10 días de antimicrobianos. Pero no mencionan manejo de cavidad peritoneal, lo cual en esta propuesta sí lo consideramos.

La clasificación sugerida muestra en este estudio que sí es posible homogeneizar las características de los hallazgos quirúrgicos, estadificarlos y nos da una pauta para decidir un manejo quirúrgico adecuado tomando en cuenta el estadio del apéndice, la cavidad peritoneal y las condiciones generales del paciente. Como se observa en las gráficas, la t de Student presenta un resultado estadístico significativo $<0.05$, lo cual nos da la pauta para sugerir esta clasificación. En la Figura 1 podemos observar que la media de días de estancia en pacientes con manejo sugerido fue de 2.8 días comparado con 13.6 días en casos sin manejo sugerido y el resultado estadístico de $p=0.04$ significativo. En la Figura 2 encontramos mayor tasa de complicaciones en los pacientes con estadios 2 sin manejo sugerido con una media de 6.8 comparada con 0.2 en los pacientes con manejo sugerido. Y en la Figura 3 observamos mayor tasa de reintervenciones en estadios 2 , 3,4 y 5 sin manejo sugerido con promedio 1.8 comparado con 0 en pacientes con manejo sugerido, con una $p=$ de 0.02 .

\section{CONCLUSIONES}

El objetivo de este nuevo sistema de clasificación fue proporcionar una forma estandarizada para permitir una estratificación del paciente más uniforme para dar pauta o guiar el manejo óptimo según el estadio, lo cual se cumplió al obtener los resultados estadísticos significativos. Por lo que esta publicación nos permite recomendar el uso de esta clasificación que, además de ser clara en la definición de la gravedad de la apendicitis aguda, se relaciona directamente con el grado de contaminación de la cavidad peritoneal, daño de tejidos adyacentes y guía el manejo quirúrgico conforme a los hallazgos transoperatorios. Ayuda a disminuir los días de estancia hospitalaria, y por ende los costos, al prevenir las complicaciones, guiarnos con los criterios generales y específicos del manejo de cavidad para evitar, complicaciones, reintervenciones y mayor riesgo de mortalidad. Facilita el proceso de sanación en la evolución del paciente, así como el proceso de enseñanzaaprendizaje de los residentes de cirugía.

Queda a consideración de todos los cirujanos, con la única intención de favorecer nuestra labor, la atención de nuestros pacientes y el proceso de enseñanza-aprendizaje de todos los residentes, al dar claridad en la gravedad para poder guiar el manejo quirúrgico con base en los hallazgos. 


\section{REFERENCIAS}

1. Campos CSF. Apendicitis aguda. En: Martínez MM Fisiopatología quirúrgica del aparato digestivo. 3ra. ed. México: Manual Moderno: 2006, 373-378.

2. Solomkin JS, Mazuski JE, Bradley JS, Rodvold KA, Goldstein EJ, Baron EJ, et al. Diagnosis and management of complicated intra-abdominal infection in adults and children: guidelines by the Surgical Infection Society and the Infectious Diseases Society of America. Clin Infect Dis. 2010; 50: 133-164.

3. Castañeda-Espinosa SD, Molina-Ramírez I, HolguínSanabria A, et al. Cambio en la clasificación macroscópica de la apendicitis. ¿Tiene algún impacto? Estudio retrospectivo en un Hospital Universitario Pediátrico. Unidad de Cirugía Pediátrica, Facultad de Medicina, Universidad Nacional de Colombia. Bogotá D.C., Colombia. Rev Fac Med. 2015; 63: 243-250.

4. Liang Mike. El apéndice. En: Brunicardi FC. Schwartz principios de cirugía. 10ª ed. México: McGraw Hill; 2015, 1241-1262.

5. Quevedo GL. Apendicitis aguda: clasificación, diagnóstico y tratamiento. Temas de actualización del Manual de procedimientos de diagnóstico y tratamiento en Cirugía General. Rev Cubana Cir. 2007; 46 (2).

6. Peranteau WH. Appendix, Meckel's, and other small bowel diverticula. En: Michael J. Zinner, MD. Maingot's abdominal operations. Twelfth Edition. EUA: McGraw Hill; 2013, 623-648.

7. Guzman-Valdivia Gómez. Una clasificación útil en apendicitis aguda. Cirugía general hospital general Núm. 1 Gabriel Mancera IMSS. Rev Gastroenterol México. 2003; 68: 261-268.

8. Saltiel MD. Apendicitis aguda. En: Morales SJL. Tratado de cirugía general. Asociación Mexicana de Cirugía General, A. C. Consejo Mexicano de Cirugía General, A.C. 3a. ed. México: Manual Moderno: 2017, 1222-1228.

9. Gomes CA, Nunes TA, Fonseca Chebli JM, Junior CS, Gomes CC. Sistema de clasificación de laparoscopia de apendicitis aguda: nueva visión para futuros ensayos. Surg Laparosc Endosc Percutan Tech. 2012; 22: 463-466.

10. Gomes CA, Massimo S, Solomon DS. Apendicitis aguda: propuesta de un nuevo sistema de clasificación integral basado en hallazgos clínicos, de imagen y laparoscópicos. Mundo J Emerg Surg. 2015; 10: 60.

11. Emil S, Laberge JM, Mikhail P, Baican L, Flageole H, Shaw K, et al. Appendicitis in children: a ten-year update of therapeutic recommendations. J Pediatr Surg. 2003; 38: 236-242.

12. Snelling $\mathrm{CMH}$, Poenaru D, Drover JW. Minimum postoperative antibiotic duration in advanced appendicitis in children: a review. Pediatr Surg Int. 2004; 20: 838-845.

13. Román PM. Capacidades y cien destrezas básicas para pensar. En: Román PM, Collarte IML. Tragaluz-aprendo a pensar. Guía Práctica para el profesor. Santiago de Chile. Arrayán Editores S.A.: 2007, 23-28.

14. Dellinger RP, Levy MM, Carlet JM, Bion J, Parker $M M$, Jaeschke $R$, et al. Surviving sepsis Campaign: international guidelines for management of severe sepsis and septic shock: 2008. Crit Care Med. 2008; 36: 296-327.
15. Conférence de Consensus - Prise en charge des péritonites communautaires. Ann Fr Anesth Reanim. 2001; 20: 344-472.

16. Nathens $A B$, Rotstein OD. Therapeutic options in peritonitis. Surg Clin North Am. 1994; 74: 677-692.

17. Parc Y, Frileux P. Reintervenciones por complicaciones infecciosas intraperitoneales postoperatorias. Tratado de técnicas quirúrgicas digestivas. Elsevier/Océano. 2013. Capítulo 3; 49-76.

18. Rotstein OR, Nathens AB. Peritonitis and intraabdominal abscesses. In: Wilmore D, Souba W editors. ACS surgery. Principles and practice. New York: Wed MD Inc; 2002, 1239-1262.

19. Dunn DL, Barke RA, Arenholz DH. The adjuvant effect of peritoneal fluid in experimental peritonitis: Mechanism and clinical implications. Ann Surg. 1984; 199: $37-43$

20. Hansbrough JF, Zapata-Sirvent RL, Cooper MI. Effects of topical anti-microbial on the human neutrophil respiratory burst. Arch Surg. 1991; 126: 603-608.

21. Rappaport WD, Holcomb M, Valente J. Antibiotic irrigation and the formation of intraabdominal adhesions. Am J Surg. 1989; 158: 435-437.

22. Nachón GFJ, Díaz TJ, Benítez OF, García GF. Lavado peritoneal transoperatorio con solución electrolizada por selectividad iónica en peritonitis secundaria. Cirujano General. 2010; 32: 11-16.

23. Koperna T, Schulz F. Prognosis and treatment of peritonitis. Do we need new scoring systems? Arch Surg. 1996; 131: 180-186.

24. Wittmann DH, Bansal N, Bergstein JM, Wallace JR, Wittmann MM, Aprahamian C. Staged abdominal repair compares favorably with conventional operative therapy for intra-abdominal infections when adjusting for prognostic factors with a logistic model. Theor Surg. 1994; 9: 201-207.

25. Kjonniksen I, Andersen BM, Sondenaa VG, Segadal L. Preoperative hair removal- a systematic literature review. AORN J. 2002; 75: 928-940.

26. Digison MB. A review of anti-septic agents for preoperative skin preparation. Plast Surg Nurs. 2007; 27: 185-189.

27. Kurz A, Sessler DI, Lenhardt R. Perioperative normothermia to reduce the incidence of surgicalwound infection and shorten hospitalization. N Engl J Med. 1996; 334: 1209-1215.

28. Sessler DI, Akca O. Nonpharmacological prevention of surgical wound infections. Clin Infect Dis. 2002; 35 : 1397-1404.

29. Hernández-Orduña J. Escala de mayor precisión para el diagnóstico de apendicitis aguda: análisis comparativo entre la escala de Alvarado, RIPASA y nueva propuesta. Cirujano General. 2019; 41: 247259.

30. Sawyer RG, Pruett TL: Wound infections. Surg Clin North Am. 1994; 74: 519-536.

Correspondencia:

Dr. Juan Hernández-Orduña

E-mail: juanhorduna@yahoo.com.mx Edublog:

https://cirugiaconcompetencias@blogspot.com/ 